\title{
ABELIAN UNIPOTENT SUBGROUPS OF FINITE ORTHOGONAL GROUPS
}

\author{
W. J. WONG \\ (Received 4 November 1980) \\ Communicated by D. E. Taylor
}

\begin{abstract}
If $G$ is the special orthogonal group $O^{+}(V)$ of a quadratic space $V$ over a finite field of characteristic $p$, and $r$ is a positive integer, we determine the abelian $p$-subgroups of largest order in $G$ whose fixed subspaces in $V$ have dimension at least $r$. In particular, we determine the abelian subgroups of largest order in a Sylow p-subgroup of $G$, extending some results obtained with different methods by Barry (1979).
\end{abstract}

1980 Mathematics subject classification (Amer. Math. Soc.): 20 G 40.

\section{Introduction}

Interest in the large abelian subgroups of a finite $p$-group $P$ goes back at least to Burnside (1912), and has more recently been stimulated by their use in the definition of the important Thompson subgroup $J(P)$, Thompson (1964). A particularly interesting case arises when $P$ is a Sylow $p$-subgroup of a group $G$ of Lie type defined over a finite field of characteristic $p$, since the study of the unipotent elements and subgroups of $G$ appears to be a key to understanding its structure and properties. If $G$ is a Chevalley group of type $A_{n}, B_{n}, C_{n}$ or $D_{n}$, the abelian subgroups of largest order in $P$ were determined by Barry (1979), applying an inductive method on a suitable parabolic subgroup of $G$, and using extensive calculations with the Chevalley commutator formula. In applications, however, $G$ often arises not as abstract group, but as a classical group of linear transformations on a vector space $V$, and then the abelian $p$-subgroups of $G$

C Copyright Australian Mathematical Society 1982 
having certain properties in this representation become of interest. In this paper we consider the case that $G$ is an orthogonal group, and study the abelian $p$-subgroups of $G$ of largest order fixing the vectors of an $r$-dimensional subspace of $V$. Our methods are again inductive, but use the geometry of the situation rather than the Chevalley formula.

We begin by explaining some necessary notation and terminology in Section 1. In Section 2, we obtain a recursion formula for the largest order of an abelian $p$-subgroup of $G$ fixing the vectors of an $r$-dimensional subspace, and we find its explicit value in Section 3. The description of the groups which occur is given in Section 4, except for a few cases in low dimensions. In Section 5 we show how our results give the largest abelian subgroups in a fixed Sylow $p$-subgroup of $G$, thus providing an alternate method to that of Barry, and completing his results for the case of the orthogonal groups. The paper closes with some remarks in Section 6.

Most of the work in this paper is independent of the value of $p$. However, it is convenient to omit the case when $p=2$ and $\operatorname{dim} V$ is odd, so as to avoid the "defective" situation in which the bilinear form on $V$ is degenerate.

\section{Notation and terminology}

We take a finite field $F$ of $q$ elements, having characteristic $p$, and consider a finite-dimensional vector space $V$ over $F$, equipped with a quadratic form. This is a map $Q: V \rightarrow F$ such that

$$
Q(a v+b w)=a^{2} Q(v)+b^{2} Q(w)+a b B(v, w),
$$

for all $v, w \in V, a, b \in F$, where $B$ is a bilinear form. In general our terminology will be similar to (but not identical with) that of Dieudonné (1955). In the following glossary, $W$ and $Z$ are subspaces of $V$, with $W \subseteq Z$.

$W^{\perp}=$ orthogonal complement of $W$ in $V$, relative to $B$.

$W_{Z}^{\perp}=W^{\perp} \cap Z$.

Radical of $W, \operatorname{rad} W=W_{W}^{\perp}$.

$W$ is defective if $\operatorname{rad} W \neq 0$.

$W$ is totally isotropic if $\operatorname{rad} W=W$.

Index of $V=$ largest dimension $\nu$ of a totally isotropic subspace.

Singular vector: $v \neq 0, Q(v)=0$.

$Q$-radical of $W, \operatorname{rad}_{Q} W=\{w \in \operatorname{rad} W \mid Q(w)=0\}$.

$W$ is degenerate if $\operatorname{rad}_{Q} W \neq 0$.

$W$ is singular if $\operatorname{rad}_{Q} W=W$.

$Q$-index of $V=$ largest dimension $\nu_{Q}$ of a singular subspace.

If $p \neq 2$, then $\operatorname{rad}_{Q} W=\operatorname{rad} W$, defective $=$ degenerate, and so on. 
We assume throughout that $V$ is nondefective of dimension $n$. Then $\nu=$ $\left(\frac{1}{2}\right)(n-1)$ if $n$ is odd, while $\nu=\left(\frac{1}{2}\right) n$ or $\nu=\left(\frac{1}{2}\right) n-1$ if $n$ is even. In the latter case we shall occasionally say $B$ is of hyperbolic type or nonhyperbolic type according as $\nu=\left(\frac{1}{2}\right) n$ or $\nu=\left(\frac{1}{2}\right) n-1$. If $p=2, B$ is an alternating form, so that $n$ must be even and $B$ is of hyperbolic type.

The orthogonal group $O(V)$ consists of all linear transformations $\sigma$ on $V$ such that $Q(\sigma v)=Q(v)$, for all $v \in V$. For technical reasons, we restrict to the special orthogonal group $O^{+}(V)$ (of index 2 in $O(V)$ ), which is the set of all $\sigma$ in $O(V)$ for which $\operatorname{dim}(\sigma-1) V$ is even, Dye (1977).

We shall say an element or subgroup of $O^{+}(V)$ fixes a subspace $X$ of $V$ if it fixes every vector in $X$. More generally, it fixes a quotient $X / Y$ of two subspaces of $V$ such that $X \supseteq Y$, if it maps every $\operatorname{coset} x+Y$ on itself $(x \in X)$.

We shall frequently use the following facts.

(a) For $W \subseteq V, \operatorname{dim} W+\operatorname{dim} W^{\perp}=\operatorname{dim} V$, and $\left(W^{\perp}\right)^{\perp}=W$.

(b) If $W$ is a singular subspace of $V$, then

$$
\bar{Q}(v+W)=Q(v) \quad\left(v \in W^{\perp}\right)
$$

defines a nondefective quadratic form $\bar{Q}$ on $W^{\perp} / W$.

(c) If an element or subgroup of $O^{+}(V)$ fixes a quotient $X / Y$ of subspaces of $V$, then it also fixes $Y^{\perp} / X^{\perp}$.

(d) The only element $\sigma$ of $\mathrm{O}^{+}(V)$ fixing a hyperplane of $V$ is the identity (since $\operatorname{dim}(\sigma-1) V \leqslant 1$ and $\operatorname{dim}(\sigma-1) V$ is even).

\section{Recursion formula}

For $0 \leqslant r \leqslant n$, we let $\mathscr{B}(V, r)$ denote the set of all abelian $p$-subgroups $A$ of $O^{+}(V)$ such that $A$ fixes some subspace of dimension $r$, and let $Q(V, r)$ be the set of all members of $\mathscr{B}(V, r)$ having largest possible order. We write the order of an element of $\mathcal{Q}(V, r)$ as $q^{f(n, r)}$. This is an incomplete notation, since, for a given $n$, there can be different quadratic forms, giving different orthogonal groups (when $n$ is even). However, it will turn out that two quadratic forms whose associated bilinear forms have the same type (hyperbolic or nonhyperbolic) give the same value of $f(n, r)$. Thus ambiguity will be avoided if it is understood that, for even dimension, the notation is always used for spaces whose bilinear forms have the same type as the space $V$ in which we are interested. We shall use such spaces in our inductive argument.

If is clear that $f(n, r)$ is a nonincreasing function of $r$, and that $f(n, n)=0$. Also $f(n, n-1)=0$, since only the identity element of $O^{+}(V)$ fixes a hyperplane. 
The following result shows that $f(n, 0)=f(n, 1)$, and also allows us to set up our basic inductive situation.

LeMma 1. If $P$ is a $p$-subgroup of $O^{+}(V), P \neq 1$, then the vectors of $V$ fixed by $P$ form a degenerate subspace. In particular, $P$ fixes some singular vector.

Proof. Suppose that the subspace $W$ of fixed vectors is nondegenerate. We have $W \neq V$, so that $W^{\perp} \neq 0$. Since $W^{\perp}$ is invariant under $P$, a counting argument (or Engel's theorem) shows that $P$ fixes a nonzero vector of $W^{\perp}$, so that $\operatorname{rad} W \neq 0$. Thus $W$ is defective, $p=2$, and $X=\operatorname{rad} W$ has dimension 1 , by Dieudonné (1955), pages 33, 34. Write $W=X \oplus Y$, where $Y$ is a nondefective subspace (possibly 0 ), and set $Z=Y^{\perp}$. Then $X \subseteq X_{Z}^{\perp} \subset Z$, and all these subspaces are invariant under $P$. If $X \neq X_{Z}^{\perp}$, then $P$ fixes some nonzero element of $X_{Z}^{\perp} / X$, so that there exists a vector $v \in X_{Z}^{\perp}, v \notin X$, such that $\sigma v \equiv v$ $(\bmod X)$, for every $\sigma \in P$. Since $v$ is orthogonal to $X, Q(\sigma v)=Q(v)+$ $Q(\sigma v-v)$, and so $Q(\sigma v-v)=0, \sigma v-v \in \operatorname{rad}_{Q} W=0$, by nondegeneracy of $W$. Thus $v \in W \cap Z=X$, a contradition. Hence $X_{Z}^{\perp}=X, \operatorname{dim} Z=2$, and $W$ is a hyperplane of $V$. Since only the identity element of $O^{+}(V)$ fixes a hyperplane, this is a contradiction, and Lemma 1 is proved.

If $x$ is a singular vector of $V$, we can choose another singular vector $y$, such that $B(x, y)=1$, by Dieudonné (1955), pages 20,33. Then $x, y$ is called a hyperbolic pair, and spans a nondefective subspace $\langle x, y\rangle$. The restriction of $B$ to the orthogonal complement $\langle x, y\rangle^{\perp}$ is of the same type as $B$.

Lemma 2. Suppose $x, y$ is a hyperbolic pair in $V$, and $Z=\langle x, y\rangle^{\perp}$, so that $V=\langle x\rangle \oplus Z \oplus\langle y\rangle$. For $\mu \in O(Z), t \in Z$, define a linear transformation $\sigma(\mu, t)$ on $V$ by

$$
\begin{aligned}
& \sigma(\mu, t) x=x, \\
& \sigma(\mu, t) z=\mu z-B(\mu z, t) x, \quad z \in Z, \\
& \sigma(\mu, t) y=y+t-Q(t) x .
\end{aligned}
$$

Then $\sigma(\mu, t)$ is an orthogonal transformation fixing $x$, and, conversely, every orthogonal transformation of $V$ fixing $x$ has the form $\sigma(\mu, t)$, for unique $\mu \in O(V)$, $t \in Z$. Multiplication of two such transformations is given by the equation

$$
\sigma(\mu, t) \sigma\left(\mu^{\prime}, t^{\prime}\right)=\sigma\left(\mu \mu^{\prime}, t+\mu t^{\prime}\right) .
$$

In particular, $\sigma(\mu, t)$ and $\sigma\left(\mu^{\prime}, t^{\prime}\right)$ commute if and only if $\mu \mu^{\prime}=\mu^{\prime} \mu$ and $(\mu-1) t^{\prime}$ $=\left(\mu^{\prime}-1\right) t$. Finally, $\sigma(\mu, t) \in O^{+}(V)$ if and only if $\mu \in O^{+}(Z)$. 
Proof. Any orthogonal transformation $\sigma$ fixing $x$ must leave $\langle x\rangle^{\perp}=\langle x\rangle \oplus$ $Z$ invariant, and so must have the form

$$
\begin{aligned}
& \sigma x=x, \\
& \sigma z=\mu z+f(z) x, \quad z \in Z, \\
& \sigma y=a y+t+b x,
\end{aligned}
$$

where $\mu: Z \rightarrow Z, f: Z \rightarrow F$ are linear maps, $t \in Z$, and $a, b \in F$. Necessary and sufficient conditions for $\sigma$ to be orthogonal are that $Q(\sigma z)=Q(z), B(\sigma z, \sigma y)=$ $0, B(\sigma x, \sigma y)=1, Q(\sigma y)=0$, for all $z \in Z$. There are equivalent to: $\mu \in O(Z)$, $f(z)=-B(\mu z, t), a=1, b=-Q(t)$, and so $\sigma=\sigma(\mu, t)$.

The multiplication formula is a straightforward calculation.

In the special case $\mu=1$, if $t \neq 0$ then $B(z, t) \neq 0$ for some $z$, and so $(\sigma(1, t)-1) V=\langle x, t\rangle$ has dimension 2. If $t=0$, then $\sigma(1,0)=1$. Hence $\sigma(1, t)$ $\in O^{+}(V)$, for all $t \in Z$. Since $\sigma(\mu, t)=\sigma(1, t) \sigma(\mu, 0), \sigma(\mu, t) \in O^{+}(V)$ if and only if $\sigma(\mu, 0) \in O^{+}(V)$. But $(\sigma(\mu, 0)-1) V=(\mu-1) Z$, and so $\sigma(\mu, 0) \in$ $O^{+}(V)$ if and only if $\mu \in O^{+}(Z)$. This proves Lemma 2 .

If $L=\left\{\sigma \in O^{+}(V) \mid \sigma x=x\right\}$, then $\sigma(\mu, t) \rightarrow \mu$ is a homomorphism $\theta: L \rightarrow$ $O^{+}(Z)$ with kernel $N=\{\sigma(1, t) \mid t \in Z\}$ isomorphic to the additive group of $Z$, and in fact $L$ is a splilt extension of $Z$ by $O^{+}(Z)$.

In applying Lemma 2, it will be convenient to use a standard notation as follows.

Basic Situation 1. The initial ingredients are a singular vector $x$, and a subspace $X$ of $V$ whose radical contains $x$. Take $y$ and $Z$ as in Lemma 2, and define the transformations $\sigma(\mu, t)$. Set $W=X \cap Z$, so that $X=\langle x\rangle \oplus W$. We note that $\sigma(\mu, t)$ fixes $X /\langle x\rangle$ if, and only if, $\mu$ fixes $W$, and $\sigma(\mu, t)$ fixes $X$ if, and only if, $\mu$ fixes $W$ and $t \in W_{Z}^{\perp}$.

We shall make reference to this situation in constructing groups $\mathrm{n} \mathscr{Q}(V, r)$. We shall also use Lemma 2 when we already have a group in $\mathcal{Q}(V, r)$. Here we have a rather more elaborate configuration, and use a standard notation as follows.

Basic Situation 2. The initial ingredient is a group $A$ in $\mathcal{Q}(V, r)$, with $A \neq 1$. By Lemma 1 , there is a degenerate $r$-dimensional subspace $X$ fixed by $A$ (possibly not the subspace of all vectors fixed by $A$ ), and a singular vector $x$ may be chosen in $\operatorname{rad} X$. From $x$ and $X$, we set up Basic Situation 1, with its notation. The homomorphism $\theta: L \rightarrow O^{+}(Z)$ shows that $|A|=|M|\left|A_{1}\right|$, where $M=A \cap N$ and

$$
A_{1}=\theta(A)=\left\{\mu \in O^{+}(Z) \mid \boldsymbol{\sigma}(\mu, t) \in A \text {, some } t\right\} .
$$

( $A_{1}$ is "essentially the same" as the group of transformations induced by $A$ on $\langle x\rangle^{\perp} /\langle x\rangle$.) If $\sigma(\mu, t) \in A$, then $t \in W_{Z}^{\perp}$. In particular, $\{t \in Z \mid \sigma(1, t) \in M\}$ 
spans a subspace $S$ of $W_{Z}^{\perp}$. Write $s=\operatorname{dim} S$, so that $s<n-r-1$. If $\sigma(\mu, t) \in$ $A, \sigma\left(1, t^{\prime}\right) \in M$, then, since $A$ is abelian, $(\mu-1) t^{\prime}=0$. Thus $A_{1}$ is an abelian $p$-subgroup of $O^{+}(Z)$ fixing both $W$ and $S$. Hence,

$$
|M| \leqslant q^{s}, \quad\left|A_{1}\right| \leqslant q^{f(n-2, d)} .
$$

where $d=\operatorname{dim}(W+S)$, and we remind the reader that $f(n-2, d)$ refers to the space $Z$, whose bilinear form has the same type as that of $V$.

We now proceed to find an upper bound for $f(n, r)$. By Lemma 1, we may assume $r \geqslant 1$, and also $n \geqslant 3$, since $O^{+}(V)$ has no nontrivial $p$-subgroup if $n \leqslant 2$, as only the identity fixes a hyperplane.

LeMma 3. Let $n \geqslant 3,1 \leqslant r \leqslant n$, and set

$$
g(n, r, s)=\left\{\begin{array}{l}
s+f(n-2, \max (r-1, s)), \quad \text { if } r<\nu, \\
s+f(n-2, \max (r-1, s+1)), \quad \text { if } r>\nu .
\end{array}\right.
$$

Then, $f(n, r) \leqslant \max \{g(n, r, s) \mid 0 \leqslant s \leqslant n-r-1\}$.

Proof. We set up Basic Situation 2. Clearly

$$
d=\operatorname{dim}(W+S) \geqslant \max (r-1, s) .
$$

If $r>\nu$, then $\operatorname{dim} W$ exceeds the index $\nu-1$ of $Z$, so that $W$ cannot be totally isotropic. Since $S \subseteq W_{Z}^{\perp}$, it follows in this case that $W \nsubseteq S$, and so

$$
d \geqslant \max (r-1, s+1) \text {. }
$$

Thus, $|A|=|M|\left|A_{1}\right| \leqslant q^{g(n, r, s)}$, for some value of $s$ between 0 and $n-r-1$. This proves Lemma 3.

To show that we have equality in Lemma 3 requires the construction of some abelian $p$-subgroups of $O^{+}(V)$. The $r$-dimensional fixed subspaces will lie in a certain family, defined as follows.

Let $\delta(V)$ be the set of all subspaces $X$ of $V$ such that (a) $X \supseteq X^{\perp}$, (b) $X \subseteq X^{\perp}$, or (c) $\operatorname{dim} X=\left(\frac{1}{2}\right) n$ and $\operatorname{dim}(\operatorname{rad} X)=\nu=\left(\frac{1}{2}\right) n-1$.

LEMMA 4. Let $r, s$ be nonnegative integers, $r+s<n, X \in \delta(V)$, and

$$
\operatorname{dim} X= \begin{cases}\max (r, s), & \text { if } r<\nu, \\ \max (r, s+1), & \text { if } r>\nu .\end{cases}
$$

Then there exist subspaces $W, S$ of $X$ of dimensions $r, s$ respectively, such that $W$ is orthogonal to $S$, and $W, S \in \mathcal{S}(V)$.

Proof. If $s+1>r>\nu$, then $s \geqslant r \geqslant \nu+1$, so that $r+s>2(\nu+1)>n$. Then we must have $s=r=\nu+1=\left(\frac{1}{2}\right) n$. Then $\operatorname{dim} X=\left(\frac{1}{2}\right) n+1, \operatorname{dim} X^{\perp}=$ $\left(\frac{1}{2}\right) n-1$, and, since $X \in \delta(V), X \supset X^{\perp}$. Take any subspace $W$ of dimension 
$\left(\frac{1}{2}\right) n$ with $X^{\perp} \subset W \subset X$, and let $S=W^{\perp}$, also of dimension $\left(\frac{1}{2}\right) n$. Then $X^{\perp} \subset S \subset X$, and $\operatorname{rad} W=\operatorname{rad} S=X^{\perp}$, so that $W, S \in \delta(V)$.

Now suppose we do not have the case $r=s=\left(\frac{1}{2}\right) n, \nu=\left(\frac{1}{2}\right) n-1$. Then $\operatorname{dim} X=\max (r, s)$, and we have symmetry between $r$ and $s$. We may assume $r>s$, so that $\operatorname{dim} X=r$, and we take $W=X$. If $X \supseteq X^{\perp}$, then $\operatorname{dim} X^{\perp}=n$ $-\operatorname{dim} X=n-r \geqslant s$, and we take $S$ to be any $s$-dimensional subspace of $X^{\perp}$. If $X \subseteq X^{\perp}$, we take $S$ to be any $s$-dimensional subspace of $X$. If $r=\left(\frac{1}{2}\right) n$, $\operatorname{dim}(\operatorname{rad} X)=\nu=\left(\frac{1}{2}\right) n-1$, then $s \neq\left(\frac{1}{2}\right) n$ by supposition, so that $s<\left(\frac{1}{2}\right) n-1$, and we take $S$ to be any $s$-dimensional subspace of $\operatorname{rad} X$. In all three cases, $S \subseteq S^{\perp}$, so that $S \in S(V)$. This proves Lemma 4 .

RECURSION FORMULA. $f(n, 0)=f(n, 1)$. If $1<r \leqslant n$, then

$$
f(n, r)=\left\{\begin{array}{l}
0, \quad \text { if } n \leqslant 2, \\
\max \{g(n, r, s) \mid 0<s<n-r-1\}, \quad \text { if } n \geq 3,
\end{array}\right.
$$

where

$$
g(n, r, s)= \begin{cases}s+f(n-2, \max (r-1, s)), & \text { if } r \leqslant \nu, \\ s+f(n-2, \max (r-1, s+1)), & \text { if } r>\nu .\end{cases}
$$

There exist an $r$-dimensional subspace $X \in \mathcal{S}(V)$ and a subgroup $A \in \mathbb{Q}(V, r)$, such that $A$ fixes $X$.

Proof. We prove this result by induction on $n$. As noted before, Lemma 1 gives $f(n, 0)=f(n, 1)$, and also $f(n, r)=0$ for $n<2$. Assume $n \geqslant 3$, and let $0<s<n-r-1$. Let $d=\max (r-1, s)$ if $r<\nu, d=\max (r-1, s+1)$ if $r>\nu$. Take a singular vector $x$ and choose $y, Z$ as in Lemma 2. Then $Z$ has dimension $n-2$, and index $v-1$ (and the same type as $V$ ). By induction, there exists a subspace $Y \in \delta(Z)$ of dimension $d$ such that $O^{+}(Z)$ has an abelian $p$-subgroup $A_{1}$ of order $q^{f(n-2, d)}$ fixing $Y$. By Lemma 4 applied to $Z$ instead of $V$, there exist subspaces $W, S$ of $Y$ of dimensions $r-1, s$ respectively, such that $W$ and $S$ are orthogonal to each other, and $W, S \in \mathcal{S}(Z)$. Then $A=\{\sigma(\mu, t) \mid \mu$ $\left.\in A_{1}, t \in S\right\}$ is an abelian $p$-subgroup of order $q^{g(n, r, s)}$ in $O^{+}(V)$, fixing the $r$-dimensional subspace $X=\langle x\rangle \oplus W$, which lies in $\delta(V)$.

Choice of the value of $s$ giving the largest value of $g(n, r, s)$, together with Lemma 3, completes the proof of the Recursion Formula.

It is easy to verify that, if the group $A_{1}$ of the proof is elementary abelian, then so is $A$. Thus the induction proof shows that $\mathcal{Q}(V, r)$ always contains at least one elementary abelian group. The descriptions obtained in Section 4 will show that in fact the groups in $\mathscr{Q}(V, r)$ are almost always elementary abelian. 
We note that the equality in the Recursion Formula implies that, in the Basic Situation 2, we have $M=\{\sigma(1, t) \mid t \in S\}$, and $A_{1} \in \mathbb{Q}\left(Z, d^{\prime}\right)$, where $d^{\prime}=$ $\max (r-1, s)$ or $\max (r-1, s+1)$ according as $r<\nu$ or $r>\nu$. Also, $f(n-2, d)=f\left(n-2, d^{\prime}\right)$, where $d=\operatorname{dim}(W+S) \geqslant d^{\prime}$.

\section{Explicit values}

For small values of $n$, calculation with the Recursion Formula gives the values of $f(n, r)$ shown in the table.

\begin{tabular}{|c|c|c|c|c|c|c|c|c|c|c|c|}
\hline & \multicolumn{11}{|c|}{$r$} \\
\hline & $n$ & 1 & 2 & 3 & 4 & 5 & 6 & 7 & 8 & 9 & 10 \\
\hline & 1 & 0 & & & & & & & & & \\
\hline & 3 & 1 & 0 & 0 & & & & & & & \\
\hline & 5 & 3 & 2 & 1 & 0 & 0 & & & & & \\
\hline & 7 & 5 & 4 & 4 & 3 & 1 & 0 & 0 & & & \\
\hline & 9 & 7 & 7 & 7 & 7 & 6 & 3 & 1 & 0 & 0 & \\
\hline & 2 & 0 & 0 & & & & & & & & \\
\hline$\nu=\left(\frac{1}{2}\right) n$ & 4 & 2 & 1 & 0 & 0 & & & & & & \\
\hline (Hyperbolic) & 6 & 4 & 3 & 3 & 1 & 0 & 0 & & & & \\
\hline & 8 & 6 & 6 & 6 & 6 & 3 & 1 & 0 & 0 & & \\
\hline & 2 & 0 & 0 & & & & & & & & \\
\hline$\nu=\left(\frac{1}{2}\right) n-1$ & 4 & 2 & 1 & 0 & 0 & & & & & & \\
\hline (Nonhyperbolic) & 6 & 4 & 3 & 2 & 1 & 0 & 0 & & & & \\
\hline & 8 & 6 & 5 & 5 & 4 & 3 & 1 & 0 & 0 & & \\
\hline & 10 & 8 & 8 & 8 & 8 & 7 & 6 & 3 & 1 & 0 & 0 \\
\hline
\end{tabular}

In each section of the table, the last row illustrates a general pattern holding for all larger values of $n$. Also, if $r>\left(\frac{1}{2}\right)$, the values of $f(n, r)$ are the triangular numbers.

THEOREM I. (a) If $r>\left(\frac{1}{2}\right) n$, then $f(n, r)=\left(\frac{1}{2}\right)(n-r)(n-r-1)$.

(b) If $n=2 m+1, r \leqslant m$, and $m \geqslant 4$, then $f(n, r)=\left(\frac{1}{2}\right) m(m-1)+1$.

(c) If $n=2 m, \nu=m, r<m$, and $m>4$, then $f(n, r)=\left(\frac{1}{2}\right) m(m-1)$.

(d) If $n=2 m, \nu=m-1$, and $m \geqslant 5$, then $f(n, r)=\left(\frac{1}{2}\right)\left(m^{2}-3 m+6\right)$

if $r<m$, and $f(n, m)=\left(\frac{1}{2}\right)\left(m^{2}-3 m+4\right)$. 
Proof. (a) We use induction on $n$, the result being true for $n<2$. Suppose $n>3$. If $0<s<n-r-1$, then $s+1<n-r<r-1$, so that $g(n, r, s)=s$ $+f(n-2, r-1)$. Thus, in the expression for $f(n, r)$ given in the Recursion Formula, the maximum value of $g(n, r, s)$ occurs (only) at $s=n-r-1$, and $f(n, r)=n-r-1+f(n-2, r-1)$. By inductive hypothesis, $f(n-2, r-1)$ $=\left(\frac{1}{2}\right)(n-r-1)(n-r-2)$. Hence, $f(n, r)=\left(\frac{1}{2}\right)(n-r)(n-r-1)$.

(b) We use induction on $m$, the result being true for $m=4$ by the table. Let $m>5$. If $s \leqslant r-1$, then $g(n, r, s)=s+f(n-2, r-1)$ is a strictly increasing function of $s$, and so $f(n, r)=\max \{g(n, r, s) \mid r-1<s<n-r-1\}$. If $r-1$ $<s<m-1$, then $g(n, r, s)=s+f(n-2, s)=s+\left(\frac{1}{2}\right)(m-1)(m-2)+1$, by inductive hypothesis, and so the maximum value of $g(n, r, s)$ for $s$ in this range is $m-1+\left(\frac{1}{2}\right)(m-1)(m-2)+1=\left(\frac{1}{2}\right) m(m-1)+1$. for $m<s<n-$ $r-1, g(n, r, s)=s+f(n-2, s)=s+\left(\frac{1}{2}\right)(n-2-s)(n-3-s)$, by (a). This is a convex function of $s$, whose maximum value on the closed interval from $m$ to $n-2$ is $m+\left(\frac{1}{2}\right)(n-2-m)(n-3-m)=\left(\frac{1}{2}\right) m(m-1)+1$. Thus, $f(n, r)$ $=\left(\frac{1}{2}\right) m(m-1)+1$, and the maximum of $g(n, r, s)$ occurs at $s=m-1, m$.

(c), (d) We omit the proof in these cases, as it is similar to that in case (b). We simply state the values of $s$ for which the maximum value of $g(n, r, s)$ occurs.

$$
\begin{aligned}
& n=2 m, \nu=m, m \geqslant 5, r \leqslant m: s=m-1 . \\
& n=2 m, \nu=m-1, m \geqslant 6, r<m: s=m-2, m-1, m . \\
& n=2 m, \nu=m-1, m>6, r=m: s=m-2, m-1 .
\end{aligned}
$$

\section{The groups}

We shall give descriptions of the groups in $Q(V, r)$ in most cases, including the "general" cases covered by Theorem I. Most of the groups occurring will be given as the group of all elements of $O^{+}(V)$ fixing some elements $x$ or subspaces $W$ or quotients $X / Y$ of subspaces of $V$. It will be convenient to use the notation exemplified by

$$
A(x, W, X / Y)=\left\{\sigma \in O^{+}(V) \mid \sigma \text { fixes } x, W \text { and } X / Y\right\} .
$$

The subspaces of fixed vectors of elements of $\mathscr{Q}(V, r)$ are usually, but not always, in the family $\delta(V)$ defined in Section 2 . We enlarge this family slightly by defining $\mathscr{X}(V)$ to be the set of all subspaces $X$ of $V$ such that (a) $X \supseteq X^{\perp}$, (b) $X \subseteq X^{\perp}$, (c) $\operatorname{dim} X=\left(\frac{1}{2}\right) n$ and $\operatorname{dim}(\operatorname{rad} X)=\nu=\left(\frac{1}{2}\right) n-1$, (d) $X$ is degenerate and $\operatorname{dim} X=n-2$, or (e) $X$ is degenerate, $\operatorname{dim} X<3, n=6$ and $\nu=2$. We shall write $\mathcal{X}_{r}(V)$ for the set of all $r$-dimensional subspaces of $V$ lying in $\mathfrak{X}(V)$. 
We shall describe the groups in $\mathcal{Q}(V, r)$ in a series of propositions, labelled in accordance with the cases of Theorem I. We shall give proofs for the propositions corresponding to cases (a) and (b), and omit proofs for the remaining cases, which use the same basic ideas although some cases require more elaborate calculations.

Of course, $\mathbb{Q}(V, r)$ contains only the identity group if $r>n-2$.

Proposition A. Let $\left(\frac{1}{2}\right) n<r \leqslant n-2$. Then

$$
\mathcal{Q}(V, r)=\left\{A(X) \mid X \in \mathcal{X}_{r}(V)\right\} .
$$

Proof. We use induction on $n$, the result being vacuously true if $n<3$. Assume $n \geqslant 4, X \in \mathcal{X}_{r}(V)$. Either $r<n-2$ and $X^{\perp} \subseteq X$, or $r=n-2$ and $X$ is degenerate. Since $\operatorname{rad}_{Q} X$ has codimension at most 1 in $\operatorname{rad} X$, we can in any case choose a nonzero vector $x$ in $\operatorname{rad}_{Q} X$, and set up Basic Situation 1 as in Section 2. Then $A(X)$ consists of all $\sigma(\mu, t)$ such that $\mu$ fixes $W$ and $t \in W_{Z}^{\perp}$. If $r=n-2$, then $W$ is a hyperplane of $Z, A(X)=\left\{\sigma(1, t) \mid t \in W_{Z}^{\perp}\right\}$ is an abelian group of order $q$, and thus $A(X) \in \mathcal{Q}(V, r)$. If $r<n-2$, then $W_{Z}^{\perp} \subset W$, since $X^{\perp} \subset X$, and so $W \in \mathfrak{X}_{r-1}(Z)$. By inductive hypothesis, the group $A_{1}$ of elements of $O^{+}(Z)$ fixing $W$ is an element of $\mathcal{Q}(Z, r-1)$. Since $W_{Z}^{\perp} \subset W$, $(\mu-1) t^{\prime}=0$, for all $\mu \in A_{1}, t^{\prime} \in W_{Z}^{\perp}$. Hence, $A(X)=\left\{\sigma(\mu, t) \mid \mu \in A_{1}, t \in\right.$ $\left.W_{Z}^{\perp}\right\}$ is abelian. A calculation of orders, using Theorem I, shows that $A(X) \in$ $\mathbb{Q}(V, r)$.

Conversely, suppose $A \in \mathbb{Q}(V, r)$, and set up Basic situation 2. As noted earlier, $M=\{\sigma(1, t) \mid t \in S\}$, and $A_{1} \in \mathcal{Q}\left(Z, d^{\prime}\right)$, where, in this case, $d^{\prime}=$ $\max (r-1, s)$. In the proof of Theorem I(a), we saw that we must have $s=n-r-1$. It follows that $S=W_{Z}^{\perp}$, and $d^{\prime}=r-1$, since $r>\left(\frac{1}{2}\right) n$. Also, we know that $d=\operatorname{dim}(W+S) \geqslant d^{\prime}$, and $f(n-2, d)=f\left(n-2, d^{\prime}\right)$. If $r<n-$ 2 , it follows from Theorem I that $d=d^{\prime}$, so that $S \subseteq W$. Setting $X=\langle x\rangle \oplus$ $W$, we have $X \in \mathcal{X}_{r}(V)$ in any case. Since $A \subseteq A(X)$, and $A(X) \in \mathcal{Q}(V, r)$, we have $A=A(X)$. This proves Proposition A.

We remark that, in Proposition A, $X$ is the subspace of all fixed vectors of $A(X)$, since $f(n, r+1)<f(n, r)$. A similar remark holds for the groups described in Propositions B1, C1, D1, D2.

Proposition B1. Let $n=2 m+1, m \geqslant 1$. Then $\mathbb{Q}(V, m)$ consists of all groups $A\left(X, X^{\perp} /\langle x\rangle\right)$, where $X \in \mathscr{X}_{m}(V)$ and $0 \neq x \in X$, 
Proof. Suppose $X \in \mathcal{X}_{m}(V)$, so that $X$ is totally isotropic, and let $0 \neq x \in$ $X$. Set up Basic Situation 1 , and let $S=W_{Z}^{\perp}$. Then $S \in \mathscr{X}_{m}(Z)$, and $A\left(X, X^{\perp} /\langle x\rangle\right)$ consists of all $\sigma(\mu, t)$ such that $\mu$ fixes $S$ and $t \in S$. By Proposition $\mathrm{A}$, the group $A_{1}$ of all $\mu$ in $O^{+}(Z)$ fixing $S$ lies in $\mathcal{Q}(Z, m)$ if $m>2$, and the same holds trivially if $m=1$. Since $(\mu-1) t^{\prime}=0$ for $\mu \in A_{1}, t^{\prime} \in S$, $A\left(X, X^{\perp} /\langle x\rangle\right)$ is abelian. A calculation of orders, using Theorem $\mathrm{I}$, shows that $A\left(X, X^{\perp} /\langle x\rangle\right) \in \mathbb{Q}(V, m)$.

Conversely, suppose $A \in \mathbb{Q}(V, m)$, and set up Basic Situation 2. In the proof of Theorem I(b), we saw that $s=m$ or $s=m-1$, provided $m>5$. This holds also when $m<5$, in the case $r=m$. Suppose first that $s=m$. Arguments as in the proof of Proposition A show that $W=S_{Z}^{\perp} \subset S$, and so $X=\langle x\rangle \oplus W \in$ $\mathscr{X}_{m}(V)$. Since $A_{1}$ fixes $S=W_{Z}^{\perp}, A$ fixes $X^{\perp} /\langle x\rangle$, so that $A \subseteq A\left(X, X^{\perp} /\langle x\rangle\right)$, and hence $A=A\left(X, X^{\perp} /\langle x\rangle\right)$

Now suppose $s=m-1$. Then $W=S \subset W_{Z}^{\perp}$, so that $X=\langle x\rangle \oplus W \in$ $\mathfrak{X}_{m}(V)$. If $m=1$, then $X=\langle x\rangle$ and $A$ automatically fixes $X^{\perp} /\langle x\rangle$, since $O^{+}\left(X^{\perp} /\langle x\rangle\right)=1$. Then $A \subseteq A\left(X, X^{\perp} /\langle x\rangle\right)$, and so $A=A\left(X, X^{\perp} /\langle x\rangle\right)$. If $m>1$, then since $A_{1} \in \mathcal{Q}(Z, m-1)$ and $A_{1}$ fixes $W$, an inductive hypothesis will show that $A_{1}$ is the subgroup of $O^{+}(Z)$ fixing $W$ and $W_{Z}^{\perp} /\left\langle x_{1}\right\rangle$, for some nonzero $x_{1}$ in $W$. We now choose $y_{1}$ in $Z$ so that $x_{1}, y_{1}$ is a hyperbolic pair, and let $Z_{1}=\left\langle x_{1}, y_{1}\right\rangle \frac{1}{Z}$, so that we have a Basic Situation 1 for $A_{1}$. For $\lambda \in O^{+}\left(Z_{1}\right)$, $t_{1} \in Z_{1}$, we define a transformation $\mu\left(\lambda, t_{1}\right)$ on $Z$ analogously to the definition of $\sigma(\mu, t)$ on $V$. Set $W_{1}=W \cap Z_{1}$. Then the elements of $A_{1}$ have the form $\mu\left(\lambda, t_{1}\right)$, where $\lambda$ fixes $\left(W_{1}\right)_{Z_{1}}^{\perp}$, and $t_{1} \in\left(W_{1}\right)_{z_{1}}^{\perp}$.

Since $\operatorname{dim} W_{1}=m-2, \operatorname{dim}\left(W_{1}\right) \frac{1}{Z_{1}}=m-1$, we can write $\left(W_{1}\right) \frac{1}{Z_{1}}=W_{1} \oplus$ $\langle u\rangle$, and so $W_{z}^{\perp}=W \oplus\langle u\rangle$, for some vector $u$. Suppose $\sigma(\mu, t), \sigma\left(\mu^{\prime}, t^{\prime}\right)$ lie in $A$, where $\mu, \mu^{\prime} \in A_{1}$, and $t, t^{\prime} \in W_{Z}^{\perp}$. Suppose $\mu=\mu\left(\lambda, t_{1}\right), \mu^{\prime}=\mu\left(\lambda^{\prime}, t_{1}^{\prime}\right)$, $t=w+c u, t^{\prime}=w^{\prime}+c^{\prime} u$, where $w, w^{\prime} \in W$, and $c, c^{\prime} \in F$. Since $A$ is abelian, $(\mu-1) t^{\prime}=\left(\mu^{\prime}-1\right) t$. A calculation shows that $c B\left(u, t_{1}^{\prime}\right)=c^{\prime} B\left(u, t_{1}\right)$. We can choose $t_{1}^{\prime}$ in $\left(W_{1}\right) \frac{1}{Z_{1}}$ such that $B\left(u, t_{1}^{\prime}\right) \neq 0$, since otherwise $\left(W_{1}\right) \frac{1}{Z_{1}}$, would be totally isotropic of dimension greater than $\left(\frac{1}{2}\right) \operatorname{dim} Z_{1}$. Use this $t_{1}^{\prime}$ with a suitable $\lambda^{\prime}$ to get an element $\mu^{\prime}$ of $A_{1}$, and choose $t^{\prime}$ so that $\sigma\left(\mu^{\prime}, t^{\prime}\right) \in A$. Then we see that $c=k B\left(u, t_{1}\right)$, where $k$ is independent of $\mu$ and $t$.

A calculation now shows that

$$
(\sigma(\mu, t)-1) u=-B\left(u, t_{1}\right)\left(x_{1}+k B(u, u) x\right) .
$$

Since $X^{\perp}=X \oplus\langle u\rangle$, and $A$ fixes $X$, we see that $A \subseteq A\left(X, X^{\perp} /\left\langle x^{\prime}\right\rangle\right)$, where $x^{\prime}=x_{1}+k B(u, u) x$, and hence $A=A\left(X, X^{\perp} /\left\langle x^{\prime}\right\rangle\right)$. This proves Proposition B1.

Proposition B2. Let $n=2 m+1, m \geqslant 4,1 \leqslant r \leqslant m$. Then $\mathbb{Q}(V, r)=$ $\mathbb{Q}(V, m)$, unless $m=4, r=1$, in which case $\mathbb{Q}(V, 1)$ consists of $\mathbb{Q}(V, 4)$ together with all groups $A\left(x,\langle x\rangle^{\perp} /\langle x\rangle\right)$, where $x$ is a singular vector of $V$. 
Proof. By Theorem I, $\mathbb{Q}(V, m) \subseteq \mathbb{Q}(V, r), r \leqslant m$. If $m=4$ and $x$ is a singular vector, take $X=\langle x\rangle^{\perp}$ and set up Basic Situation 1. Then $A\left(x,\langle x\rangle^{\perp} /\langle x\rangle\right)=\{\sigma(1, t) \mid t \in Z\}$ is an abelian group of order $q^{7}$, and so lies in $\mathbb{Q}(V, 1)$.

Conversely, suppose $A \in \mathbb{Q}(V, r)$, and set up Basic Situation 2. We now introduce a new method for studying $A$. For $\mu \in A_{1}$, we set

$$
C_{\mu}=\{t \in Z \mid \sigma(\mu, t) \in A\} .
$$

For $\sigma(\mu, t)$ to fix $W, t$ must lie in $W_{Z}^{\perp}$, so that $C_{\mu} \subseteq W_{Z}^{\perp}$. The equation $\sigma\left(1, t^{\prime}\right) \sigma(\mu, t)=\sigma\left(\mu, t+t^{\prime}\right)$ shows that $C_{\mu}$ is a coset of $S$ in $W_{Z}^{\perp}$, and so we have a map

$$
\gamma: A_{1} \rightarrow W_{Z}^{\perp} / S
$$

given by $\gamma(\mu)=C_{\mu}$. Since $A_{1}$ fixes $S$ and $W, W_{Z}^{\perp} / S$ is an $A_{1}$-module, and the equation $\sigma(\mu, t) \sigma\left(\mu^{\prime}, t^{\prime}\right)=\sigma\left(\mu \mu^{\prime}, t+\mu t^{\prime}\right)$ shows that $\gamma$ is a crossed homomorphism. The group $A$ is determined by $A_{1}$ and $\gamma$.

The "kernel" of $\gamma$,

$$
K=\left\{\mu \in A_{1} \mid C_{\mu}=S\right\},
$$

is a subgroup of $A_{1}$, and the image of $\gamma$ has cardinality $\left|A_{1}: K\right|$. Since $W_{Z}^{\perp} / S$ has order $q^{n-r-s-1}$, a lower bound for $|K|$ is given by

$$
|K|>\left|A_{1}\right| / q^{n-r-s-1}>\left|A_{1}\right| / q^{n-s-2} .
$$

Theorem I can now be used to find an upper bound on the dimension of the subspace

$$
T=\bigcap_{\mu \in K} \operatorname{ker}(\mu-1)
$$

of vectors of $Z$ fixed by $K$. If $\mu \in K, t \in S$, the equation $(\mu-1) t^{\prime}=\left(\mu^{\prime}-1\right) t$ $=0$ for all $\mu^{\prime} \in A_{1}, t^{\prime} \in C_{\mu^{\prime}}$ shows that every $C_{\mu^{\prime}}$ is contained in $T$, so that the image of $\gamma$ is in $T / S$. Thus a new lower bound for $|K|$ is given by

$$
|K| \geqslant\left|A_{1}\right| / q^{\operatorname{dim} T-s} \text {. }
$$

Application of Theorem I gives a new upper bound on $\operatorname{dim} T$, and so on, and thus we obtain information on the image of $\gamma$.

In our present case, we have $s=m$ or $s=m-1$, unless $m=4$ and $r=1$, when we may also have $s=7$.

Suppse first that $s=m$. Then $A_{1} \in \mathbb{Q}(Z, m)$, and $A_{1}$ fixes $S$. By Proposition A, $S \in \mathfrak{X}_{m}(Z)$ and $A_{1}$ consists of all elements of $O^{+}(Z)$ which fix $S$. We have $\left|A_{1}\right|=q^{\left(\frac{1}{2}\right)(m-1)(m-2)}$. If $m>4$, the method outlined above leads to the successive bounds $|K| \geqslant q^{\left(\frac{1}{2}\right)(m-1)(m-4)}, \operatorname{dim} T<m+1,|K|>q^{\left(\frac{1}{2}\right)(m-1)(m-2)-1}, \operatorname{dim} T$ $\leqslant m$. Thus $T=S$, all $C_{\mu}=S$, and so

$$
A=\left\{\sigma(\mu, t) \mid \mu \in A_{1}, t \in S\right\} .
$$

Now $A$ fixes $\langle x\rangle \oplus S_{Z}^{\perp}$, and so $A \in \mathbb{Q}(V, m)$. 
The same argument works when $s=m=4$, if $K \neq 1$. Suppose $K=1$, so that $\gamma$ is a bijection of $A_{1}$ on $Z / S$ (and $r=1$ ). Since $A_{1}$ fixes $S, A_{1}$ also fixes $Z / S_{Z}^{+}$. Also, $S_{Z}^{\perp}$ is singular, since $S_{Z}^{\perp} \subset S$ and $p \neq 2$. Thus, if $\mu \in A_{1}, z \in Z$, we have $Q((\mu-1) z)=0$, so that $Q(\mu z)=Q(z)+B((\mu-1) z, z)$, and hence $B((\mu-1) z, z)=0$. The map taking $z_{1}, z_{2}$ to $B\left((\mu-1) z_{1}, z_{2}\right)$ is therefore an alternating bilinear form on $Z$, and so is skew-symmetric. In particular, if $\mu, \mu^{\prime} \in A_{1}, t \in C_{\mu}, t^{\prime} \in C_{\mu^{\prime}}$, then

$$
B\left((\mu-1) t, t^{\prime}\right)=-B\left((\mu-1) t^{\prime}, t\right)=-B\left(\left(\mu^{\prime}-1\right) t, t\right)=0 .
$$

Since $\gamma$ is surjective, $t^{\prime}$ can be any element of $Z$, and so $(\mu-1) t=0$, since $Z$ is nondegenerate. If $\mu, \mu^{\prime} \in A_{1}, t \in C_{\mu}, t^{\prime} \in C_{\mu^{\prime}}$, then $t+\mu t^{\prime} \in C_{\mu \mu^{\prime}}$, and so

$$
\left(\mu \mu^{\prime}-1\right)\left(t+\mu t^{\prime}\right)=0 \text {. }
$$

Since $\mu \mu^{\prime}=\mu^{\prime} \mu, \mu t=t, \mu^{\prime} t^{\prime}=t^{\prime}$, we obtain

$$
\left(\mu^{\prime}-1\right) t+\mu(\mu-1) t^{\prime}=0 .
$$

Since $\left(\mu^{\prime}-1\right) t=(\mu-1) t^{\prime}$, this gives

$$
\left(\mu^{2}-1\right) t^{\prime}=0 .
$$

Since $t^{\prime}$ can be any element of $Z$, this implies that $\mu^{2}=1$, and so $\mu=1$, since $p$ is odd. Thus $A_{1}=1$, a contradiction. This disposes of the case $s=m$.

Next suppose $s=m-1$. Then $A_{1} \in \mathbb{Q}(Z, m-1)$, and $A_{1}$ fixes $S$. By Proposition B1, $S$ is totally isotropic, and there exists a nonzero $x_{1}$ in $S$ such that $A_{1}$ consists of all elements of $O^{+}(Z)$ fixing both $S$ and $S_{Z}^{+} /\left\langle x_{1}\right\rangle$. Arguing as before with the kernel $K$ of the crossed homomorphism $\gamma$, and its fixed subspace $T$, we find that $\operatorname{dim} T \leqslant m$, unless $m=4$ and $\gamma$ is a bijection. If $T=S$, then $A=\left\{\sigma(\mu, t) \mid \mu \in A_{1}, t \in S\right\}$ fixes $\langle x\rangle \oplus S$, and so $A \in \mathbb{Q}(V, m)$.

Suppose that $\operatorname{dim} T=m$. Then $|K|>q^{\left(\frac{1}{2}\right)(m-1)(m-2)}$. Since $K$ fixes $T$, it follows from Theorem I that $|K|=q^{\left(\frac{1}{2}\right)(m-1)(m-2)}, K \in \mathbb{Q}(Z, m)$, and $T_{Z}^{\perp} \subset T$ by Proposition A. Then, $T_{Z}^{\perp}=\{z \in T \mid Q(z)=0\} \supseteq S$, and so $T_{Z}^{\perp}=S$. Hence $S$ is fixed by all $\sigma(\mu, t)$ with $t \in C_{\mu}$, so that $A$ fixes $\langle x\rangle \oplus S$, and $A \in \mathbb{Q}(V, m)$.

If $m=4$ and $\gamma$ is a bijection, then $(\mu-1)^{2} z \in\left\langle x_{1}\right\rangle$, for all $\mu \in A_{1}, z \in Z$, since $A_{1}$ fixes $Z / S_{Z}^{\perp}$ and $S_{Z} /\left\langle x_{1}\right\rangle$. Hence $Q\left(\mu^{2} z-2 \mu z+z\right)=0$. Expanding the left side and using the fact that $\mu$ is orthogonal, we find

$$
B((\mu-1)(\mu-3) z, z)=0,
$$

so that the bilinear map on $Z$ taking $z_{1}, z_{2}$ to $B\left((\mu-1)(\mu-3) z_{1}, z_{2}\right)$ is alternating. If $\mu, \mu^{\prime} \in A_{1}, t \in C_{\mu}, t^{\prime} \in C_{\mu^{\prime}}$, we set $z=(\mu-3) t, z^{\prime}=\left(\mu^{\prime}-3\right) t^{\prime}$. Then

$$
B\left((\mu-1)(\mu-3) z, z^{\prime}\right)=-B\left((\mu-1)(\mu-3) z^{\prime}, z\right) .
$$


Since $\mu$ commutes with $\mu^{\prime}$, and $(\mu-1) t^{\prime}=\left(\mu^{\prime}-1\right) t$, we have $(\mu-1)(\mu-3) z^{\prime}$ $=\left(\mu^{\prime}-1\right)\left(\mu^{\prime}-3\right) z$, and so

$$
B\left((\mu-1)(\mu-3) z, z^{\prime}\right)=-B\left(\left(\mu^{\prime}-1\right)\left(\mu^{\prime}-3\right) z, z\right)=0 .
$$

Let $H=\left\langle x_{1}\right\rangle \frac{\perp}{Z}$. Since $A_{1}$ fixes $S_{Z}^{\perp}\left\langle x_{1}\right\rangle$, it also fixes $H / S$, and so, if $t^{\prime} \in H$, $\left(\mu^{\prime}-3\right) t^{\prime} \equiv-2 t^{\prime}(\bmod S)$. Since $\gamma$ is surjective, $t^{\prime}$ can be any element of $H$, and so

$$
(\mu-1)(\mu-3)^{2} t=(\mu-1)(\mu-3) z \in H_{Z}^{1}=\left\langle x_{1}\right\rangle .
$$

Since $(\mu-1)^{2}$ maps $Z$ into $\left\langle x_{1}\right\rangle$, and $(\mu-1)(\mu-3)^{2}=(\mu-1)^{3}-4(\mu-1)^{2}$ $+4(\mu-1)$, we see that $(\mu-1) t \in\left\langle x_{1}\right\rangle$. The same calculation as in the case $s=m$, taken $\bmod \left\langle x_{1}\right\rangle$, shows that $(\mu-1) Z \subseteq\left\langle x_{1}\right\rangle$, for all $\mu \in A_{1}$. Since $\mu \in O^{+}(Z)$, we must have $\mu=1$, so that $A_{1}=1$, a contradiction. This completes the case $s=m-1$.

Finally, suppose that $m=4, r=1, s=7$. Then $A_{1}=1$, and $A=\{\sigma(1, t) \mid t \in$ $Z\}$, so that $A=A\left(x,\langle x\rangle^{\perp} /\langle x\rangle\right)$. This proves Proposition B2.

As previously mentioned, we omit the proofs of the remaining propositions in this section.

Proposition C1. Let $n=2 m, m \geqslant 2, \nu=m$. Then,

$$
\mathscr{Q}(V, m)=\left\{A(X) \mid X \in \mathscr{X}_{m}(V)\right\} .
$$

Before stating the next result, we define some groups that occur in the case $n=8, p=2$. First suppose $\nu_{Q}=4$, and take a singular subspace $X$ of dimension 4 in $V$. Let $x$ be a nonzero element of $X$, and set up Basic Situation 1. If $v, w \in W=X \cap Z$, define a linear map $\theta(v, w)$ on $Z$ by

$$
\theta(v, w) z=B(z, v) w+B(z, w) v .
$$

Then $v, w \rightarrow \theta(v, w)$ is an alternating bilinear map of $W \times W$ into End $Z$, and so we have a linear map of $\wedge W$ into End $Z$ such that $v \wedge w \rightarrow \theta(v, w)$. Every element of $\wedge^{2} W$ is decomposable, $\operatorname{dim} \wedge^{2} W=3$, and one can check that the image in End $Z$ has dimension 3. If $\mu(v, w)=\theta(v, w)+1$, then $\mu(v, w) \in$ $O^{+}(Z)$, and $v \wedge w \rightarrow \mu(v, w)$ is an isomorphism of the additive group $\wedge w$ with a subgroup $A_{1}$ of $O^{+}(Z)$ fixing $W$, so that $A_{1} \in \mathbb{Q}(Z, 3)$.

Now take a nonzero element $e$ of the 1-dimensional exterior power 3 $\wedge(X /\langle x\rangle)$. Since $X /\langle x\rangle$ is naturally isomorphic with $W$, we can consider $e$ as an element of $\wedge^{3} W$. Then $e$ defines a natural isomorphism of $\wedge^{2} W$ with the dual space $W^{*}$ of $W$, while the bilinear form $B$ defines an isomorphism of $W^{*}$ 
with $Z / W$. We obtain an isomorphism $\tau: \stackrel{2}{\wedge} W \rightarrow Z / W$, such that $\tau(v \wedge w)=$ $t+W$, where, for $u \in W, u \wedge v \wedge w=B(t, u) e$. Clearly $t$ is orthogonal to $\langle v, w\rangle$.

Given $\mu, \mu^{\prime} \in A_{1}$, we can write $\mu=\mu(v, w), \mu^{\prime}=\mu\left(v, w^{\prime}\right)$, since any two 2-dimensional subspaces of $W$ intersect in a line. If $\tau(v \wedge w)=t+W$, $\tau\left(v \wedge w^{\prime}\right)=t^{\prime}+W$, then $(\mu-1) t^{\prime}=B\left(t^{\prime}, w\right) v,\left(\mu^{\prime}-1\right) t=B\left(t, w^{\prime}\right) v$. Since

$$
B\left(t^{\prime}, w\right) e=w \wedge v \wedge w^{\prime}=w^{\prime} \wedge v \wedge w=B\left(t, w^{\prime}\right) e
$$

we have $(\mu-1) t^{\prime}=\left(\mu^{\prime}-1\right) t$. If now we set, for $\mu=\mu(v, w) \in A_{1}, C_{\mu}=$ $\tau(v \wedge w)$, then

$$
A(x, X /\langle x\rangle ; e)=\left\{\sigma(\mu, t) \mid \mu \in A_{1}, t \in C_{\mu}\right\}
$$

is an abelian subgroup of order $q^{6}$ in $O^{+}(V)$, fixing $x$. There are $q-1$ such groups for each pair $X, x$, depending on the choice of $e$ :

Now suppose $n=8, q=2$, and let $X$ be a totally isotropic subspace of dimension 4 in $V$ which is not singular. Let $x$ be a nonzero element of $\operatorname{rad}_{Q} X$. Again set up Basic Situation 1. Take a basis $x_{1}, x_{2}, x_{3}$ of $W=X \cap Z$ such that $x_{1}, x_{2} \in \operatorname{rad}_{Q} W$ (and $Q\left(x_{3}\right)=1$ ), and extend to a basis $x_{1}, x_{2}, x_{3}, y_{1}, y_{2}, y_{3}$ of $Z$, where $B\left(x_{i}, y_{j}\right)=0$ when $i \neq j$, and $Q\left(y_{1}\right)=Q\left(y_{2}\right)=0$. The group $A_{1}$ of elements of $O^{+}(Z)$ fixing $W$ consists of all linear transformations $\mu(a, b, c)$, where $a, b, c \in F, \mu(a, b, c)$ fixes $W$, and

$$
\begin{aligned}
& \mu(a, b, c) y_{1}=y_{1}+a x_{1}+b x_{2}+a x_{3}, \\
& \mu(a, b, c) y_{2}=y_{2}+b x_{1}+c x_{2}+c x_{3}, \\
& \mu(a, b, c) y_{3}=y_{3}+a x_{1}+c x_{2} .
\end{aligned}
$$

For $\mu=\mu(a, b, c)$, set $C_{\mu}=a\left(y_{2}+y_{3}\right)+b y_{3}+c\left(y_{1}+y_{3}\right)+W$. Then,

$$
\left\{\sigma(\mu, t) \mid \mu \in A_{1}, t \in C_{\mu}\right\}
$$

is an abelian subgroup of order $2^{6}$ in $O^{+}(V)$ fixing $x$, which, for uniformity of notation, we again denote as $A(x, X /\langle x\rangle ; e)$, where now the superfluous symbol $e$ may be thought of as the unique nonzero element of $\wedge^{3}(X /\langle x\rangle)$. This group is independent of the choice of basis made in its definition.

Proposition C2. Let $n=2 m, m \geqslant 4, \nu=m$, and $1<r \leqslant m$. Then $\mathcal{Q}(V, r)=$ $\mathbb{Q}(V, m)$, unless $m=4$ and $r=1$. If $m=4$, and either $p \neq 2$, or $q>2$ and $\nu_{Q}=3$, then $\mathscr{Q}(V, 1)$ consists of $\mathscr{Q}(V, 4)$ together with all groups $A\left(x,\langle x\rangle^{\perp} /\langle x\rangle\right)$, where $x$ is a singular vector of $V$. If $m=4, p=2$, and either $q=2$ or $\nu_{Q}=4$, then $\mathbb{Q}(V, 1)$ consists of $\mathbb{Q}(V, 4)$, the groups $A\left(x,\langle x\rangle^{\perp} /\langle x\rangle\right)$, and the groups $A(x, X /\langle x\rangle ; e)$, where $X$ is a totally isotropic 4-dimensinal subspace of $V, 0 \neq x \in X, 0 \neq e \in \bigwedge^{3}(X /\langle x\rangle)$, and $X$ is singular if $q>2$. 
It remains to deal with the nonhyperbolic case.

Proposition D1. Let $n=2 m, m \geqslant 2, \nu=m-1$. Then, $\mathbb{Q}(V, m)$ consists of all groups $A\left(X, X^{\perp} /\langle x\rangle\right)$, where $X \in \mathfrak{X}_{m}(V)$ and $0 \neq x \in \operatorname{rad} X$.

The next case, $n=2 m, v=m-1, r=m-1$, is more complicated. Let $X$ be a totally isotropic $(m-1)$-dimensional subspace of $V$. Then $X^{\perp} / X$ is an anisotropic plane relative to the bilinear form $\bar{B}$ inherited from $B$. Suppose $\zeta$ is a self-adjoint linear transformation on $X^{\perp} / X$, that is,

$$
\bar{B}(\bar{u}, \zeta \bar{v})=\bar{B}(\zeta \bar{u}, \bar{v}),
$$

for $\bar{u}, \bar{v} \in X^{\perp} / X$. If $x_{1}, x_{2} \in X, \bar{u} \in X^{\perp} / X$, then

$$
\tau \bar{v}=\bar{B}(\bar{u}, \bar{v}) x_{1}+\bar{B}(\bar{u}, \zeta \bar{v}) x_{2}
$$

defines a linear map $\tau=\tau\left(x_{1}, x_{2}, \bar{u}\right)$ of $X^{\perp} / X$ into $X$. Let $\mathcal{L}\left(x_{1}, x_{2}, \zeta\right)$ be the set of all $\tau\left(x_{1}, x_{2}, \bar{u}\right)$, as $\bar{u}$ ranges over $X^{\perp} / X$. We write $A\left(X, X^{\perp} / X ; x_{1}, x_{2}, \zeta\right)$ for the group of all elements $\sigma$ of $O^{+}(V)$ such that $\sigma$ fixes $X$ and $X^{\perp} / X$, and the map of $X^{\perp} / X$ into $X$ induced by $\sigma-1$ lies in $\varrho\left(x_{1}, x_{2}, \zeta\right)$.

Proposition D2. Let $n=2 m, \nu=m-1$. If $m \geqslant 4$, then $Q(V, m-1)$ consists of all groups $A\left(X, X^{\perp} / X ; x_{1}, x_{2}, \zeta\right)$, where $X \in \mathcal{X}_{m-1}(V), x_{1}, x_{2}$ are a linearly independent pair in $X$, and $\zeta$ is a self-adjoint linear transformation on $X^{\perp} / X$. If $m=3$, then $\mathcal{Q}(V, 2)$ consists of all such groups $A\left(X, X^{\perp} / X ; x_{1}, x_{2}, \zeta\right)$ for totally isotropic $X$ in $\mathscr{X}_{2}(V)$, together with all groups $A\left(X, X^{\perp} /\langle x\rangle\right)$ for subspaces $X$ in $\mathcal{X}_{2}(V)$ which are not totally isotropic, $0 \neq x \in \operatorname{rad} X$.

The groups $A\left(X, X^{\perp} / X ; x_{1}, x_{2}, \zeta\right)$ can be described more simply in some cases. If $\zeta$ is a scalar multiple of $1, \zeta=c 1$, then $A\left(X, X^{\perp} / X ; x_{1}, x_{2}, \zeta\right)=$ $A\left(X, X^{\perp} /\langle x\rangle\right)$, where $x=x_{1}+c x_{2}$. Suppose then that $\zeta$ is not a scalar multiple of 1 . We note that $\mathcal{L}\left(x_{1}, x_{2}, \zeta\right)=\mathcal{L}\left(x_{1}-a^{-1} b x_{2}, a^{-1} x_{2}, a \zeta+b\right)$, if $a, b \in F, a \neq 0$. Since $\zeta$ satisfies its characteristic equation, of degree 2 , we can therefore arrange to obtain $\zeta^{2}=c 1, c \in F$.

If $c$ is a square, we can get $\zeta^{2}=1$, and there exists an orthogonal basis $\bar{v}_{1}, \bar{v}_{2}$ of $X^{\perp} / X$ such that $\zeta \bar{v}_{1}=\bar{v}_{1}, \zeta \bar{v}_{2}=-\bar{v}_{2}$. If $\bar{u} \in X^{\perp} / X, \tau=\tau\left(x_{1}, x_{2}, \bar{u}\right)$, then

$$
\tau \bar{v}_{1}=\bar{B}\left(\bar{u}, \bar{v}_{1}\right)\left(x_{1}+x_{2}\right), \quad \tau \bar{v}_{2}=\bar{B}\left(\bar{u}, \bar{v}_{2}\right)\left(x_{1}-x_{2}\right) .
$$

If $\left\langle\bar{v}_{1}\right\rangle=Y / X$, where $Y$ is a subspace of $V$ containing $X$, then $Y \in \mathfrak{X}_{m}(V)$, $\left\langle\bar{v}_{2}\right\rangle=Y^{\perp} / X$. Set $y=x_{1}+x_{2}, y^{\prime}=x_{1}-x_{2}$, linearly independent elements of $X=Y \cap Y^{\perp}$. Then $A\left(X, X^{\perp} / X ; x_{1}, x_{2}, \zeta\right)=A\left(Y /\langle y\rangle, Y^{\perp} /\left\langle y^{\prime}\right\rangle\right)$. 
If $c$ is a nonsquare, there exists a basis $\bar{v}_{1}, \bar{v}_{2}$ of $X^{\perp} / X$, with $\bar{B}\left(\bar{v}_{2}, \bar{v}_{2}\right)=$ $c \bar{B}\left(\bar{v}_{1}, \bar{v}_{1}\right)$, such that $\zeta \bar{v}_{1}=\bar{v}_{2}, \zeta \bar{v}_{2}=c \bar{v}_{1}$. If $\bar{u} \in X^{\perp} / X, \tau=\tau\left(x_{1}, x_{2}, \vec{u}\right)$, then

$$
\begin{aligned}
& \tau \bar{v}_{1}=\bar{B}\left(\bar{u}, \bar{v}_{1}\right) x_{1}+\bar{B}\left(\bar{u}, \bar{v}_{2}\right) x_{2}, \\
& \tau \bar{v}_{2}=\bar{B}\left(\bar{u}, \bar{v}_{2}\right) x_{1}+c \bar{B}\left(\bar{u}, \bar{v}_{1}\right) x_{2} .
\end{aligned}
$$

Thus $\mathcal{E}\left(x_{1}, x_{2}, \zeta\right)$ consists of all the linear combinations of the two maps $\tau_{1}, \tau_{2}$, where $\tau_{1} \bar{v}_{1}=x_{1}, \tau_{1} \bar{v}_{2}=c x_{2}, \tau_{2} \bar{v}_{1}=x_{2}, \tau_{2} \bar{v}_{2}=x_{1}$. It does not seem possible to give a simpler description of $A\left(X, X^{\perp} / X ; x_{1}, x_{2}, \zeta\right)$ in this case. Of course, we can take $c$ to be any prescribed nonsquare.

Proposition D3. Let $n=2 m, \nu=m-1, m \geqslant 5,1 \leqslant r \leqslant m-1$. Then $\mathbb{Q}(V, r)=\mathbb{Q}(V, m-1)$, unless $m=5$ and $r=1$. If $m=5$, then $\mathbb{Q}(V, 1)$ consists of $\mathbb{Q}(V, 4)$ together with all groups $A\left(x,\langle x\rangle^{\perp} /\langle x\rangle\right)$, where $x$ is a singular vector of $V$.

This completes the description of the groups in $\mathcal{Q}(V, r)$ in all but a few cases of low dimension, where similar results can be found. For example, if $n<7$, or if $n=8$ and $\nu=3$, then $Q(V, 1)$ consists of all groups $A\left(x,\langle x\rangle^{\perp} /\langle x\rangle\right)$, where $x$ is a singular vector. Not covered by our results are the three cases when $\nu=3$, $r=2$.

If we define the "unipotency class" of $A$ to be the least number $c=c(A)$ such that $\left(\sigma_{1}-1\right)\left(\sigma_{2}-1\right) \cdots\left(\sigma_{c}-1\right)=0$, for all $\sigma_{1}, \sigma_{2}, \ldots, \sigma_{c} \in A$, it can be verified that $c(A) \leqslant 3$ for all the groups $A$ occurring in this section, except when $n=8, p=2$, and $A=A(x, X /\langle x\rangle ; e)$ as in Proposition $\mathrm{C} 2$, in which case $c(A)=4$. If $p$ is odd, then it follows that $A$ is elementary abelian, since $\sigma^{p}-1=(\sigma-1)^{p}=0, \sigma \in A$. If $p=2$, it still holds that $A$ is elementary abelian, with the single exception when $n=8, q=2$, and $A=A(x, X /\langle x\rangle ; e)$ with $X$ not singular, in which case $A$ has exponent 4.

\section{The Sylow group}

We shall use our results to determine the abelian subgroups of largest order in a given Sylow $p$-subgroup $P$ of $O^{+}(V)$. We first give a description of $P$.

A sequence of singular subspaces of $V$,

$$
0=W_{0} \subset W_{1} \subset W_{2} \subset \cdots \subset W_{k},
$$

such that $\operatorname{dim} W_{i}=i$, is called a singular flag of $V$, of length $k$, and defines a subgroup $P\left(W_{0}, W_{1}, \ldots, W_{k}\right)$ of $O^{+}(V)$, consisting of all elements which fix all 
$W_{i} / W_{i-1}(1 \leqslant i \leqslant k)$ and $W_{k}^{\perp} / W_{k}$. Such an element $\sigma$ also fixes all $W_{i-1}^{\perp} / W_{i}^{\perp}$, so that $\sigma-1$ maps each subspace of the chain

$$
0=W_{0} \subset W_{1} \subset \cdots \subset W_{k} \subseteq W_{k}^{\perp} \subset \cdots \subset W_{1}^{\perp} \subset W_{0}^{\perp}=V
$$

into the preceding one. Thus, $(\sigma-1)^{2 k+1}=0$, so that $\sigma^{p^{a}}-1=(\sigma-1)^{p^{a}}=0$, for $p^{a} \geqslant 2 k+1$, and so $\sigma$ is a $p$-element. Thus $P\left(W_{0}, W_{1}, \ldots, W_{k}\right)$ is a $p$-group.

If $P$ is a Sylow $p$-subgroup of $O^{+}(V)$, then

$$
P=P\left(W_{0}, W_{1}, \ldots, W_{k}\right),
$$

for a singular flag of length $k=\left[\left(\frac{1}{2}\right)(n-1)\right]$. This may be seen by induction on $n$. It is clearly true if $n \leqslant 2$, since then $P=1$. Assume $n \geqslant 3$. By Lemma $1, P$ fixes a singular line $W_{1}$. Then $W_{1}^{\perp} / W_{1}$ inherits a nondefective quadratic form from $Q$, by remark (b) of Section 1 , and $P$ induces a $p$-subgroup $\bar{P}$ of $O^{+}\left(W_{1}^{\perp} / W_{1}\right)$. By induction, $\bar{P}$ lies in the $p$-group defined by a singular flag of $W_{1}^{\perp} / W_{1}$ of length $k-1$. The preimage of this flag in $W_{1}^{\perp}$ gives a singular flag of length $k$ in $V$, for which $P \subseteq P\left(W_{0}, W_{1}, \ldots, W_{k}\right)$, and thus $P=$ $P\left(W_{0}, W_{1}, \ldots, W_{k}\right)$.

Since $\operatorname{dim} W_{k}^{\perp} / W_{k} \leqslant 2, O^{+}\left(W_{k}^{\perp} / W_{k}\right)$ is a $p^{\prime}$-group, and so $P$ can also be described as the set of all $p$-elements of $O^{+}(V)$ fixing all $W_{i} / W_{i-1}(1<i<k)$.

For each type of group $A$ in $\mathbb{Q}(V, 1)$, we determine the conditions under which $A$ is contained in a given Sylow $p$-subgroup $P$. Usually $A \in \mathbb{Q}(V, v)$, where $\nu$ is the index of $V$, and we begin with this case.

Lemma 5. Suppose $A \in \mathbb{Q}(V, v)$, and assume that $\nu \geqslant 3$ if $n$ is even. Let $X$ be the subspace of all vectors fixed by $A$, and $Y$ a subspace of codimension at least 2 in $X$. Then

$$
\left\{v \in Y^{\perp} \mid(\sigma-1) v \in Y \text {, all } \sigma \in A\right\} \subseteq X^{\perp} .
$$

Proof. Under the conditions of the lemma, $X$ is totally isotropic of dimension $\nu$, by Propositions B1, C1, D2. There is a nondegenerate subspace $Z$ such that $Y^{\perp}=Y \oplus Z$, and $\operatorname{dim} Z=n-2 \operatorname{dim} Y>4$. Now $X=Y \oplus(X \cap Z)$, $\operatorname{dim}(X \cap Z)=\nu-\operatorname{dim} Y$, so that, if $X^{\prime}=(X \cap Z)_{Z}^{\perp}$, then $\operatorname{dim} X^{\prime}=r$, where $r=n-\operatorname{dim} Y-\nu$. We note that $r \leqslant \operatorname{dim} Z-2$, and $r>\left(\frac{1}{2}\right) \operatorname{dim} Z$ if $\nu<$ $\left(\frac{1}{2}\right) n$, while $r=\left(\frac{1}{2}\right) \operatorname{dim} Z$ if $\nu=\left(\frac{1}{2}\right) n$. If $B=\left\{\sigma \in O^{+}(Z) \mid \sigma\right.$ fixes $\left.X^{\prime}\right\}$, then $B \in \mathcal{Q}(Z, r)$, by Propositions $\mathrm{A}$ and $\mathrm{Cl}$, and $X^{\prime}$ is the space of all vectors in $Z$ fixed by $B$. By defining the action of $B$ on $Z^{\perp}$ to be the identity, we consider $B$ to be a subgroup of $O^{+}(V)$. Then $B$ fixes $X^{\perp}=Y \oplus X^{\prime}$, so that $B \subseteq A$, by the description of $A$ given in Propositions B1, Cl, D2.

Suppose now that $v \in Y^{\perp}$, and $(\sigma-1) v \in Y$, all $\sigma \in A$. Then $v=y+z$, $y \in Y, z \in Z$, and $(\sigma-1) z=(\sigma-1) v \in Y \cap Z=0$, for all $\sigma \in B$. Hence $z \in X^{\prime}$, so that $v \in Y \oplus X^{\prime}=X^{\perp}$. This proves Lemma 5 . 
Lemma 6. Let $P=P\left(W_{0}, W_{1}, \ldots, W_{k}\right)$ be a Sylow p-subgroup of $O^{+}(V)$, where $k=\left[\left(\frac{1}{2}\right)(n-1)\right]$, and let $A \in \mathbb{Q}(V, v)$.

(a) If $n$ is odd, $A=A\left(X, X^{\perp} /\langle x\rangle\right)$ as in Proposition B1, then $A \subseteq P$ if and only if either $X=W_{k}$, or $x \in W_{k-1} \subset X$.

(b) If $n$ is even, $\nu=\left(\frac{1}{2}\right) n, n \geqslant 6, A=A(X)$ as in Proposition C1, then $A \subseteq P$ if and only if $X \supset W_{k}$.

(c) If $n$ is even, $\nu=\left(\frac{1}{2}\right) n-1, n \geqslant 8, A=A\left(X, X^{\perp} / X ; x_{1}, x_{2}, \zeta\right)$ as in Proposition D2, then $A \subseteq P$ if and only if $X=W_{k}$, or $x_{1}, x_{2} \in W_{k-1} \subset X$, or $x_{1}+c x_{2} \in W_{k-1} \subset X$ and $\zeta=c 1$.

Proof. We consider the three cases in turn. In each case, $X$ is a maximal totally isotropic subspace (of dimension $\nu$ ).

(a) Suppose $A \subseteq P$. if $W_{i} \subset X, i \leqslant k-2$, then Lemma 5 shows that $W_{i+1} \subseteq$ $X^{\perp}$, and hence $W_{i+1} \subseteq X$, since $W_{i+1}+X$ is a totally isotropic subspace containing $X$. By induction, $W_{k-1} \subseteq X$, and so $W_{k-1} \subset X \subset X^{\perp} \subset W_{k-1}^{\perp}$. If $X \neq W_{k}$, then $A$ fixes the distinct lines $X / W_{k-1}, W_{k} / W_{k-1}$ in the 3-dimensional space $W_{k-1}^{\perp} / W_{k-1}$, and so $A$ fixes $W_{k-1}^{\perp} / W_{k-1}$. (Here we have used remarks (b), (d) of Section 1). Thus $A$ fixes $X^{\perp} / W_{k-1}$. Since $A$ fixes $X^{\perp} /\langle x\rangle$ but does not fix $X^{\perp}$, it follows that $x \in W_{k-1}$.

Conversely, if $X=W_{k}$, then $A$ consists of $p$-elements fixing all $W_{i} / W_{i-1}$ $(1<i \leqslant k)$, and so $A \subseteq P$. If $x \in W_{k-1} \subset X$, so that $W_{k-1} \subset X \subset X^{\perp} \subset$ $W_{k-1}^{\perp}$, then $A$ fixes the hyperplane $X^{\perp} / W_{k-1}$ of the 3-dimensional space $W_{k-1}^{\perp} / W_{k-1}$, and so $A$ fixes $W_{k-1}^{\perp} / W_{k-1}$. In particular, $A$ fixes $W_{k} / W_{k-1}$ and $W_{k}^{\perp} / W_{k}$, as well as all $W_{i} / W_{i-1}, 1 \leqslant i<k-1$, so that $A \subseteq P$.

(b) If $A \subseteq P$, the same argument as in (a) shows that $X \supset W_{k}$. Conversely, if $X \supset W_{k}$, then $A$ consists of $p$-elements fixing all $W_{i} / W_{i-1}$, and so $A \subseteq P$.

(c) Suppose $A \subseteq P$. As in case (a), $W_{k-1} \subset X$. If $X \neq W_{k}$, then $W_{k} \nsubseteq X^{\perp}$, for else $X+W_{k}$ would be a totally isotropic subspace properly containing $X$. Since $X^{\perp}$ is a hyperplane of $W_{k-1}^{\perp}, W_{k-1}^{\perp}=X^{\perp}+W_{k}$. Since $A$ fixes $W_{k} / W_{k-1}$ and $X^{\perp} / X$, it fixes $W_{k-1}^{\perp} / X$. By remarks (a) and (c) of Section $1, A$ also fixes $X^{\perp} / W_{k-1}$. Since the image of $X^{\perp}$ under $\{\sigma-1 \mid \sigma \in A\}$ is $\left\langle x_{1}+c x_{2}\right\rangle$ if $\zeta=c 1$ and $\left\langle x_{1}, x_{2}\right\rangle$ if $\zeta$ is not a scalar multiple of 1 , we obtain $x_{1}+c x_{2} \in$ $W_{k-1}$ or $x_{1}, x_{2} \in W_{k-1}$ accordingly.

The converse is proved by the same argument as in (a). This proves Lemma 6.

Lemma 7. Let $P=P\left(W_{0}, W_{1}, \ldots, W_{k}\right)$ be a Sylow p-subgroup of $O^{+}(V)$, and $A=A\left(x,\langle x\rangle^{\perp} /\langle x\rangle\right)$, where $x$ is a singular vector. Then $A \subseteq P$ if and only if $\langle x\rangle=W_{1}$. 
Proof. With the notation of Lemma 2, $A$ consists of all $\sigma(1, t), t \in Z$, and we check easily that $\langle x\rangle$ consists of all vectors fixed by $A$. If $A \subseteq P$, it follows that $\langle x\rangle=W_{1}$. Conversely, if $\langle x\rangle=W_{1}$, then $A$ fixes $W_{1}$ and $W_{1}^{\perp} / W_{1}$, and so $A \subseteq P$, since $W_{k}^{\perp} \subseteq W_{1}^{\perp}$.

Lemma 8. Let $n=8, \nu=4, p=2$, and let $P=P\left(W_{1}, W_{1}, W_{2}, W_{3}\right)$ be a Sylow 2-subgroup of $O^{+}(V)$. Suppose $A=A(x, X /\langle x\rangle ; e)$ as in Proposition C2. Then $A \subseteq P$ if and only if $\langle x\rangle=W_{1}$ and $X \supset W_{3}$.

Proof. Suppose $A \subseteq P$. As in Lemma $7,\langle x\rangle=W_{1}$. The group of transformations induced on $\langle x\rangle^{\perp} /\langle x\rangle$ by $A$ is an element of $\mathscr{Q}\left(\langle x\rangle^{\perp} /\langle x\rangle, 3\right)$ contained in the Sylow 2-subgroup of $O^{+}\left(\langle x\rangle^{\perp} /\langle x\rangle\right)$ defined by the sequence $W_{1} / W_{1} \subset W_{2} / W_{1} \subset W_{3} / W_{1}$. By Lemma $6(\mathrm{~b})$, the subspace of fixed points $X /\left\langle x_{1}\right\rangle=X / W_{1}$ contains $W_{3} / W_{1}$, and so $X \supset W_{3}$.

Conversely, if $\langle x\rangle=W_{1}$ and $X \supset W_{3}$, then since $A$ fixes $x$ and $X /\langle x\rangle, A$ consists of 2-elements fixing all $W_{i} / W_{i-1}$, so that $A \subseteq P$. This proves Lemma 8 .

TheOREM II. Let $P=P\left(W_{0}, W_{1}, \ldots, W_{k}\right)$ be a Sylow p-subgroup of $O^{+}(V)$, where $k=\left[\left(\frac{1}{2}\right)(n-1)\right]$, and let $\mathscr{Q}(P)$ be the set of all abelian subgroups of largest possible order in $P$. Write

$$
f(q, r, 1)=\left(q^{r}-1\right) /(q-1), f(q, r, 2)=\left(q^{r}-1\right)\left(q^{r}-q\right) /(q-1) .
$$

(a) If $n$ is odd and $n \geqslant 11$, then $\mathcal{Q}(P)$ consists of all the groups $A\left(X, X^{\perp} /\langle x\rangle\right)$, where $X$ is a maximal totally isotropic subspace of $V, 0 \neq x \in X$, and either $X=W_{k}$ or $x \in W_{k-1} \subset X$. There are $f(q, k, 1)+q f(q, k-1,1)$ such groups. If $n=9$, then $Q(P)$ consists of these groups $A\left(X, X^{\perp} /\langle x\rangle\right)$ together with one additional group $A\left(W_{1}, W_{1}^{\perp} / W_{1}\right)$.

(b) If $n$ is even, $v=\left(\frac{1}{2}\right) n$, and $n>10$, then $\mathcal{Q}(P)$ consists of all the groups $A(X)$, where $X$ is a maximal totally isotropic subspace of $V$ containing $W_{k}$. There are 2 such groups if $p \neq 2, q+1$ if $p=2$. If $n=8$, and either $p \neq 2$, or $q>2$ and $\nu_{Q}=3$, then $\mathcal{Q}(P)$ consists of these groups $A(X)$ together with one additional group $A\left(W_{1}, W_{1}^{\perp} / W_{1}\right)$. If $n=8, p=2$, and either $\nu_{Q}=4$ or $q=2$, then $\mathbb{Q}(P)$ consists of the groups $A(X)$, the group $A\left(W_{1}, W_{1}^{\perp} / W_{1}\right)$, and

(i) $2(q-1)$ groups $A\left(W_{1}, X / W_{1} ; e\right)$, where $X$ is a singular 4-dimensional subspace containing $W_{3}$, in the case that $q>2$ and $\nu_{Q}=4$; or

(ii) 3 groups of the form $A\left(W_{1}, X / W_{1}\right.$; e), where $X$ is a totally isotropic 4-dimensional subspace containing $W_{3}$, in the case that $q=2$.

(c) If $n$ is even, $\nu=\left(\frac{1}{2}\right) n-1$, and if $n>12$, then $\mathcal{Q}(P)$ consists of $f(q, k, 1)+$ $\left(q^{2}+\left(\frac{1}{2}\right) q-\frac{1}{2}\right) f(q, k, 2)$ groups $A\left(X, X^{\perp} / X ; x_{1}, x_{2}, \zeta\right)$, where $X=W_{k}$, and $q^{2} f(q, k-1,1)+q^{2}\left(q^{2}+\left(\frac{1}{2}\right) q-\frac{1}{2}\right) f(q, k-1,2)$ groups $A\left(X, X^{\perp} / X\right.$; $\left.x_{1}, x_{2}, \zeta\right)$, where $X$ is a maximal totally isotropic subspace different from $W_{k}$, 
containing $W_{k-1}$, and $x_{1}, x_{2} \in W_{k-1}$, or $x_{1}+c x_{2} \in W_{k-1}$ and $\zeta=c 1$. If $n=$ 10 , then $Q(P)$ consists of these groups together with one additional group $A\left(W_{1}, W_{1}^{\perp} / W_{1}\right)$.

(d) If $n \leqslant 7$, or if $n=8$ and $\nu=3$, then $\mathcal{Q}(P)$ consists of the single group $A\left(W_{1}, W_{1}^{\perp} / W_{1}\right)$.

Proof. By Lemma $1, \mathbb{Q}(P) \subseteq \mathbb{Q}(V, 1)$. The descriptions of the groups in $\mathbb{Q}(P)$ follow directly from the descriptions of $\mathbb{Q}(V, 1)$ in Section 4 , together with Lemmas $6,7,8$. We outline the calculations needed to determine the numbers of groups in $\mathbb{Q}(P)$.

If $n$ is odd, the number of groups $A\left(X, X^{\perp} /\langle x\rangle\right)$ with $X=W_{k}$ is the number of choices of the line $\langle x\rangle$ in $X$, which is $f(q, k, 1)$. A maximal totally isotropic subspace $X$ containing $W_{k-1}$ corresponds to an isotropic line in the 3-dimensional space $W_{k-1}^{\perp} / W_{k-1}$. Choosing an orthogonal basis for $W_{k-1}^{\perp} / W_{k-1}$, we see by Dickson (1958), page 48, that there are $q+1$ such lines. For each of the $q$ subspaces $X$ different from $W_{k}$, we can choose the line $\langle x\rangle$ in $W_{k-1}$ in $f(q, k-1,1)$ ways. Hence there are $q f(q, k-1,1)$ further groups $A\left(X, X^{\perp} /\langle x\rangle\right)$, and the enumeration in (a) follows.

If $n$ is even, $\nu=\left(\frac{1}{2}\right) n$, then a maximal totally isotropic subspace $X$ containing $W_{k}$ corresponds to an isotropic line in the 2-dimensional space $W_{k}^{\perp} / W_{k}$. If $p \neq 2$, there are 2 such lines, while all $q+1$ lines are isotropic if $p=2$. If $n=8$, $p=2, q>2, \nu_{Q}=4$, the singular 4-dimensional subspaces $X$ containing $W_{3}$ correspond to the singular lines in the 2-dimensional space $W_{3}^{\perp} / W_{3}$; there are 2 such lines. Each $X$ gives $q-1$ groups $A\left(W_{1}, X / W_{1} ; e\right)$. If $n=8, q=2$, there are 3 totally isotropic 4-dimensional subspaces $X$ containing $W_{3}$, corresponding to the 3 lines in $W_{3}^{\perp} / W_{3}$. Each $X$ gives a single group $A\left(W_{1}, X / W_{1} ; e\right)$. This completes the enumeration for case (b).

If $n$ is even, $\nu=\left(\frac{1}{2}\right) n-1$, consider the number of groups $A\left(X, X^{\perp} / X\right.$; $\left.x_{1}, x_{2}, \zeta\right)$ for which $X=W_{k}$. If $\zeta$ is a scalar multiple of the identity, $\zeta=c 1$, the number of such groups is the number of lines $\left\langle x_{1}+c x_{2}\right\rangle$ in $X$, which is given by $f(q, k, 1)$. If $\xi$ is not a scalar multiple of the identity, but $\zeta^{2}=c 1$ where $c$ is a square, then we have $A\left(Y /\langle y\rangle, Y^{\perp} /\left\langle y^{\prime}\right\rangle\right)$, where $Y$ corresponds to a line in the 2-dimensional space $X^{\perp} / X$, and $y, y^{\prime}$ form a linearly independent pair in $X$. There are $q+1$ choices of $Y$, and $f(q, k, 2)$ choices for the pair of lines $\langle y\rangle$, $\left\langle y^{\prime}\right\rangle$. Since each choice of $Y$ gives the same groups as $Y^{\perp}$, we obtain $\left(\frac{1}{2}\right)(q+1) f(q, k, 2)$ groups in this case. Finally, if $\zeta^{2}=c 1$, where $c$ is a fixed nonsquare, then for a given nonzero $\bar{v}_{1}$ in $X^{\perp} / X$, there are $q+1$ ways of choosing $\bar{v}_{2}$ in $X^{\perp} / X$ such that $\bar{B}\left(\bar{v}_{2}, \bar{v}_{2}\right)=c \bar{B}\left(\bar{v}_{1}, \bar{v}_{1}\right)$, by Dickson (1958), page 46, each giving a single $\zeta$ with $\zeta^{2}=c 1, \zeta \bar{v}_{1}=\bar{v}_{2}$. There are $(q-1)^{2} f(q, k, 2)$ choices for the linearly independent pair $x_{1}, x_{2}$, with a pair which is a multiple of 
$x_{1}, x_{2}$ by a single nonzero scalar giving the same group. Hence the number of groups in this case is $(q+1)(q-1) f(q, k, 2)$, so that in all we have $f(q, k, 1)+$ $\left(q^{2}+\left(\frac{1}{2}\right) q-\frac{1}{2}\right) f(q, k, 2)$ groups $A\left(X, X^{\perp} / X ; x_{1}, x_{2}, \zeta\right)$ with $X=W_{k}$. If $X \neq$ $W_{k}$, and $X$ corresponds to an isotropic line in the 4-dimensional space $W_{k-1}^{\perp} / W_{k-1}$, there are $q^{2}$ choices for $X$, by Dickson (1958), page 47. For each such $X$ an enumeration similar to the above holds, and we obtain the total number as in (c).

The last case (d) follows immediately from Lemma 7 and the remark following Proposition D3. This completes the proof of Theorem II.

It can be checked that our results agree with those of Barry (1979) for Chevalley groups of types $B_{n}$ and $D_{n}$, as well as covering the groups of twisted type ${ }^{2} D_{n}$.

It is easy to check which of the groups in $\mathscr{Q}(P)$ are normal in $P$. We state the result without proof.

THEOREM III. In Theorem II, let $\mathfrak{Q}_{N}(P)$ denote the set of groups in $\mathscr{Q}(P)$ which are normal in $P$.

(a) If $n$ is odd and $n \geqslant 11$, then $\mathbb{Q}_{N}(P)$ consists of the single group $A\left(W_{k}, W_{k}^{\perp} / W_{1}\right)$. If $n=9$, then $\mathbb{Q}_{N}(P)$ consists of this group together with the additional group $A\left(W_{1}, W_{1}^{\perp} / W_{1}\right)$.

(b) If $n$ is even, $\nu=\left(\frac{1}{2}\right) n, n>8$, then $\mathbb{Q}_{N}(P)=\mathbb{Q}(P)$.

(c) If $n$ is even, $\nu=\left(\frac{1}{2}\right) n-1, n>12$, then $\mathbb{Q}_{N}(P)$ consists of the single group $A\left(W_{k}, W_{k}^{\perp} / W_{1}\right)$. If $n=10$, then $\mathbb{Q}_{N}(P)$ consists of this group together with the additional group $A\left(W_{1}, W_{1}^{\perp} / W_{1}\right)$.

(d) If $n<7$, or if $n=8$ and $\nu=3$, then $\mathbb{Q}_{N}(P)=\mathbb{Q}(P)$, consisting of the single group $A\left(W_{1}, W_{1}^{\perp} / W_{1}\right)$.

In particular, $\mathfrak{Q}_{N}(P)$ is always nonempty, that is, at least one of the abelian subgroups of largest order in $P$ is normal in $P$.

\section{Remarks}

(a) If $F$ is taken to be an algebraically closed field instead of a finite field, so that $O^{+}(V)$ is an algebraic group, our methods determine the closed abelian unipotent subgroups of largest dimension $f(n, r)$ in $O^{+}(V)$, fixing the vectors of an $r$-dimensional subspace.

(b) For the other finite classical groups, the abelian unipotent subgroups fixing an $r$-dimensional subspace can be studied by our methods. In the case of the general linear groups, this was done by Goozeff (1970). The cases of the 
symplectic and unitary groups $G$ are more complicated, as the group of transformations in $G$ fixing an isotropic vector is the semidirect product of a lowerdimensional group with a unipotent group which is not abelian, but nilpotent of class 2 . We hope to deal with these cases in a later paper.

(c) Results of this paper (for the case $n=8$ ), together with some properties of Cayley algebras, have been applied in current work of the author and William Higgins to find the abelian unipotent subgroups of largest order in finite Chevalley groups of type $E_{6}$.

\section{References}

M. J. J. Barry (1979), 'Large abelian subgroups of Chevalley groups,' J. Austral. Math. Soc. Ser. A 27, 59-87.

W. Burnside (1912), 'On some properties of groups whose orders are powers of primes,' Proc. London Math. Soc. (2) 11, 225-245.

L. E. Dickson (1958), Linear groups (Dover, New York).

J. Dieudonné (1955), La géométrie des groupes classiques (Springer-Verlag, Berlin).

R. H. Dye (1977), 'A geometric characterization of the special orthogonal groups and the Dickson invariant,' J. London Math. Soc. (2) 15, 472-476.

J. T. Goozeff (1970), 'Abelian p-subgroups of the general linear group,' J. Austral. Math. Soc. 11, 257-259.

J. G. Thompson (1964), 'Normal p-complements for finite groups,' J. Algebra 1, 43-46.

Department of Mathematics

University of Notre Dame

Notre Dame, Indiana 46556

U.S.A. 